\title{
Superconductivity in doped inversion-symmetric Weyl semimetals
}

\author{
Tao Zhou, ${ }^{1,2, *}$ Yi Gao, ${ }^{3}$ and Z. D. Wang ${ }^{2, \dagger}$ \\ ${ }^{1}$ College of Science, Nanjing University of Aeronautics and Astronautics, Nanjing 210016, China \\ ${ }^{2}$ Department of Physics and Center of Theoretical and Computational Physics, The University of Hong Kong, \\ Pokfulam Road, Hong Kong, China \\ ${ }^{3}$ Department of Physics and Institute of Theoretical Physics, Nanjing Normal University, Nanjing, 210023, China
}

(Received 8 October 2015; revised manuscript received 11 February 2016; published 16 March 2016)

\begin{abstract}
We study theoretically the superconductivity in doped Weyl semimetals with an inversion symmetry based on the Bogoliubov-de Gennes equations. In principle, the two superconducting states, i.e., the zero momentum BCS-like pairing and the finite momentum Fulde-Ferrell-Larkin-Ovchinnikov (FFLO) pairing, are competing in this kind of systems. From the self-consistent calculation and the free-energy analysis, we propose that the BCS-type state may be the ground state. The competition between these two pairing states is studied in detail through normal state Fermi surface and the finite-energy spectral functions. We also study the physical properties and address the Majorana Fermions excitation in these two superconducting states, respectively.
\end{abstract}

DOI: 10.1103/PhysRevB.93.094517

\section{INTRODUCTION}

In the past decade, there has been significant interest in topological phases of condensed matter [1]. In fully gapped systems, the nontrivial topological phase is defined by a quantized topological invariant, which implies the appearance of the edge states on the boundary of the system. Recently, research has also been extended to the topological gapless systems [2,3]. In particular, the Weyl semimetal is such a representative topological fermionic gapless one [2], which has pairs of gapless points (Weyl nodes) in the bulk spectrum and Fermi arcs on the boundaries, even for disordered ones [4]. An idea of Weyl semimetal in condensed matter system was first proposed theoretically that it may be realized in a class of pyrochlore iridates [2]. Recently, it was predicted that it may be realized in noncentrosymmetric transition metal monophosphides (including TaAs, TaP, NbAs, and $\mathrm{NbP}$ ) based on the first principle calculation [5,6]. Soon after the theoretical prediction, the indications of Weyl fermions in TaAs [7,8], NbAs [9], TaP [10,11], and NbP [12], have been reported experimentally. Another kind of Weyl semimetal is the $\mathrm{WX}_{2}$ family material $\left(\mathrm{WTe}_{2}\right.$ and $\left.\mathrm{WoTe}_{2}\right)[13,14]$. Very interestingly, it was revealed that $\mathrm{WoTe}_{2}$ material is a superconductor with the maximum transition temperature at $8.2 \mathrm{~K}$ under pressure [15], providing us with a potential platform to study the interplay between the topology and superconductivity in Weyl semimetal systems.

Generally, the superconducting state should be the ground state of a metal via the pairing interaction mediated by the phonon or other bosons. Thus, it is understandable to observe the superconductivity in Weyl semimetals. Actually, the superconductivity in Weyl semimetal systems has been studied before [16-26]. Theoretically, the ground state of the Weyl-type superconductors is still to be confirmed yet. It was proposed that a finite momentum pairing state [FuldeFerrell-Larkin-Ovchinnikov (FFLO) state] is favored over the uniform zero momentum pairing (BCS-type pairing) [17], while an odd-parity superconductivity is proposed by later

\footnotetext{
*tzhou@nuaa.edu.cn

†zwang@hku.hk
}

studies [20,24]. It was proposed that the FFLO state is favored for local interaction while the BCS state wins out for nonlocal interaction [20]. Very recently, it was indicated that the BCS state is the ground state for an inversion symmetric doped Weyl semimetals [24]. Note that all of the above studies are merely based on a free energy analysis. The gap magnitude is self-consistently determined while the form of the gap functions is preset. To our knowledge, full self-consistent studies about the superconducting pairing in this kind of system are still awaited. Also, for both BCS and FFLO states, there exist some exotic features, such as the crossed flat bands in the uniform BCS-type pairing state [25] and the spacetime supersymmetry in the FFLO-type pairing state [26]. Thus, it is timely and of fundamental interest to study the competition of these two superconducting states in more detail and clarify what kind of state is the genuine ground state.

Another important issue in topological superconducting systems is the excitations of the Majorana Fermions (MFs), which can in principle help us to realize nonabelian statistics and have potential applications in topological quantum computation [27]. Generally, in a bulk gapped topological superconductor, the MFs naturally appear at the system boundaries. While for the Weyl superconductors, the BCS-type pairing state is bulk gapless, different from usual topological superconductors. And so far, the numerical identification for MFs in topological FFLO states is also lacking. Thus, it is insightful to identify the MFs numerically in possible superconducting states in Weyl semimetals.

In this paper, motivated by the above considerations, we study numerically the ground state and the MFs in the Weyl superconductors from the lattice-type model with the inversion symmetry. Following Refs. [17,24], we here consider the superconductivity in doped Weyl semimetals. While different from their studies, which are based on the free-energy analysis, we here perform a self-consistent study based on the the Bogoliubov-de Gennes (BdG) equations. Our numerical results reveal that the BCS pairing state is favorable. The competition between the BCS state and the FFLO state is studied in detail through the normal state Fermi surface and the spectral functions. The physical properties in these two superconducting states are also investigated. The MFs in both the BCS state and the FFLO state are studied numerically. 
The rest of the paper is organized as follows. In Sec. II, we introduce the model and work out the formalism. In Sec. III, we perform numerical calculations and discuss the obtained results. Finally, we give a brief summary in Sec. IV.

\section{MODEL AND FORMALISM}

We start from a model Hamiltonian including the normal state term and the interaction term,

$$
H=H_{t}+H_{e}
$$

The normal state term $H_{t}$ is a general two-band lattice-type Weyl model, given by

$$
\begin{aligned}
H_{t}= & -\sum_{\mathbf{i} \alpha \sigma} \sigma t_{\alpha}\left(c_{\mathbf{i} \sigma}^{\dagger} c_{\mathbf{i}+\hat{\alpha} \sigma}+\text { H.c. }\right)+\sum_{\mathbf{i} \sigma}(\sigma h-\mu) c_{\mathbf{i} \sigma}^{\dagger} c_{\mathbf{i} \sigma} \\
& +\sum_{\mathbf{i}}\left(i \lambda c_{\mathbf{i} \uparrow}^{\dagger} c_{\mathbf{i}+\hat{x} \downarrow}+i \lambda c_{\mathbf{i} \downarrow}^{\dagger} c_{\mathbf{i}+\hat{x} \uparrow}+\right.\text { H.c. } \\
& \left.+\lambda c_{\mathbf{i} \uparrow}^{\dagger} c_{\mathbf{i}+\hat{y} \downarrow}-\lambda c_{\mathbf{i} \downarrow}^{\dagger} c_{\mathbf{i}+\hat{y} \uparrow}+\text { H.c. }\right),
\end{aligned}
$$

with $\mathbf{i}=(x, y, z)$ representing a site on the three-dimensional cubic lattice. $\hat{\alpha}=\hat{x}, \hat{y}$, or $\hat{z}$ represents the base vector along $x, y$, or $z$ direction.

The possible superconducting pairings have been summarized in Ref. [17] based on the symmetry classification, among which the $s$-wave symmetry is suggested when only the on-site interactions are considered. The $s$-wave pairing appears to be the most common superconducting symmetry in nature. Usually the phonon-mediated superconductors possess the $s$-wave pairing symmetry. Also in the cold-atom systems, the $s$-wave pairing has also been realized [28]. Therefore, in the present work, it is reasonable to assume the superconducting pairing to be an $s$-wave one. The interaction term $H_{e}$ is considered as the on-site attractive interaction term, expressed by

$$
H_{e}=-V \sum_{\mathbf{i}} n_{\mathbf{i} \uparrow} n_{\mathbf{i} \downarrow}
$$

The above attractive interaction can be decoupled to the $s$-wave superconducting Hamiltonian by defining the on-site mean-field pairing order parameter $\Delta_{\mathbf{i i}}=V\left\langle c_{\mathbf{i} \uparrow} c_{\mathbf{i} \downarrow}\right\rangle$. Thus, the interaction term may be written as the superconducting pairing term, with

$$
H_{e}=H_{\mathrm{SC}}=\sum_{\mathbf{i}}\left(\Delta_{\mathrm{ii}} c_{\mathbf{i} \uparrow}^{\dagger} c_{\mathbf{i} \downarrow}^{\dagger}+\text { H.c. }\right)+\frac{\sum_{\mathbf{i}} \Delta_{\mathbf{i i}}^{2}}{V} .
$$

The above Hamiltonian can be diagonalized by solving the Bogoliubov-de Gennes (BdG) equations,

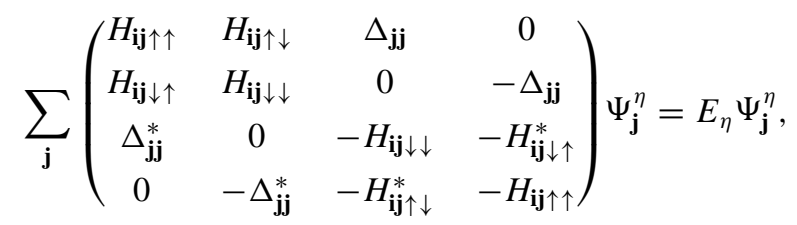

where $\Psi_{\mathbf{j}}^{\eta}=\left(u_{\mathbf{j} \uparrow}^{\eta}, u_{\mathbf{j} \downarrow}^{\eta}, v_{\mathbf{j} \downarrow}^{\eta}, v_{\mathbf{j} \uparrow}^{\eta}\right)^{\mathrm{T}} . H_{\mathbf{i} \mathbf{j} \sigma \sigma}$ and $H_{\mathbf{i j} \sigma \bar{\sigma}}(\sigma \neq \bar{\sigma})$ are obtained from Eq. (2).
The superconducting free energy, defined as $F=$ $-k_{B} T \ln Z$, can be expressed as

$$
F=k_{B} T \sum_{\eta} \ln \left[1-f\left(E_{\eta}\right)\right]-\mu N+\frac{\sum_{\mathbf{i}} \Delta_{\mathbf{i i}}^{2}}{V},
$$

with $f\left(E_{\eta}\right)$ being the Fermi distribution function. Then the site-averaged superconducting condensation energy $G$ can be obtained, with

$$
G=\frac{F-F_{0}}{N},
$$

where $F_{0}$ is normal state free energy, which is obtained by setting $\Delta_{\mathrm{ii}} \equiv 0$ in Eqs. (5) and (6). $N$ is the number of the lattice sites.

The superconducting order parameter $\Delta_{\mathbf{j j}}$ is calculated selfconsistently,

$$
\Delta_{\mathbf{j} \mathbf{j}}=\frac{V}{2} \sum_{\eta} u_{\mathbf{j} \uparrow}^{\eta} v_{\mathbf{j} \downarrow}^{\eta *} \tanh \left(\frac{E_{\eta}}{2 k_{B} T}\right) .
$$

The edge states of the system may be studied with a cylindrical geometry, i.e., considering the periodic boundary condition along the $x$ direction. Thus, the Hamiltonian can be reduced to a quasi-two-dimensional one by a partial Fourier transformation,

$$
c_{\underline{\mathbf{i}} \sigma}\left(k_{x}\right)=\frac{1}{\sqrt{L_{x}}} \sum_{x} c_{\underline{\mathbf{i}} \sigma} e^{i k_{x} x},
$$

where $L_{x}$ is the period of the lattice along the $x$ direction, and $\underline{\mathbf{i}}=(y, z)$ represents a site in the reduced $y z$ plane. As a result, the normal state Hamiltonian $H_{t}$ may be rewritten as

$$
\begin{aligned}
H_{t}= & -\sum_{k_{x} \underline{\mathbf{i}} \alpha \sigma} \sigma t_{\alpha}\left[c_{\underline{\mathbf{i}} \sigma}^{\dagger}\left(k_{x}\right) c_{\underline{\mathbf{i}}+\hat{\alpha} \sigma}\left(k_{x}\right)+\text { H.c. }\right] \\
& +\sum_{k_{x} \underline{\mathbf{i} \sigma}}\left(\sigma h-2 \sigma t_{x} \cos k_{x}-\mu\right) c_{\underline{\mathbf{i}} \sigma}^{\dagger}\left(k_{x}\right) c_{\underline{\mathbf{i}} \sigma}\left(k_{x}\right) \\
& +\sum_{k_{x} \underline{\mathbf{i}}}\left[\lambda c_{\underline{\mathbf{i}} \uparrow}^{\dagger}\left(k_{x}\right) c_{\underline{\mathbf{i}}+\hat{y} \downarrow}\left(k_{x}\right)-\lambda c_{\underline{\mathbf{i}} \downarrow}^{\dagger}\left(k_{x}\right) c_{\underline{\mathbf{i}}+\hat{y} \uparrow}\left(k_{x}\right)\right. \\
& \left.+2 \lambda \sin k_{x} c_{\underline{\mathbf{i}} \uparrow}^{\dagger}\left(k_{x}\right) c_{\underline{\mathbf{i}} \downarrow}\left(k_{x}\right)+\text { H.c. }\right] .
\end{aligned}
$$

In the superconducting state, in the mean-field level, both uniform BCS-type and FFLO-type solutions are in principle possible when the spin-polarized term exists. Usually, the FFLO modulation is suppressed and the BCS state is the ground state with a strong spin-orbital interaction [29]. In the present model, a strong spin-flip hopping term is considered in the $x$ direction, which could suppress the FFLO modulation along this direction. Thus, it is reasonable to consider the superconducting order parameter being uniform along the $x$ direction. This is qualitatively consistent with the normal state Fermi surface analysis in doped Weyl semimetals. Generally, the Fermi surface should consist of two disconnected Fermi pockets around the pair of Weyl points [17]. In principle, two competing pairing states are possible, i.e., the interpocket BCS pairing and the intrapocket FFLO pairing. Note that for both pairing states the net momentum of the Cooper pair along the $k_{x}$ direction keeps zero (see Fig. 1 in Ref. [17]). That is, the 
superconducting pairing term can be written as

$$
H_{\mathrm{SC}}=\sum_{\underline{\mathbf{i}}}\left[\Delta_{\underline{\mathbf{i}}} c_{\underline{\mathbf{i}} \uparrow}^{\dagger}\left(k_{x}\right) c_{\underline{\mathbf{i}} \downarrow}^{\dagger}\left(-k_{x}\right)+\text { H.c. }\right]+N_{x} \frac{\sum_{\underline{\mathbf{i}}} \Delta_{\underline{\mathbf{i}}}^{2}}{V} .
$$

Here $N_{x}$ represents the number of lattice sites along $x$ direction. The $k_{x}$ dependent $\mathrm{BdG}$ equation can be obtained from Eqs. (10) and (11), with the formalism similar to Eq. (5). The order parameter $\Delta_{\mathbf{j j}}$ is calculated as

$$
\Delta_{\underline{\mathbf{j}} \underline{\mathbf{j}}}=\frac{V}{2 N_{x}} \sum_{k_{x} \eta} u_{\underline{\mathbf{j}} \uparrow}^{\eta}\left(k_{x}\right) v_{\underline{\mathbf{j}} \downarrow}^{\eta *}\left(-k_{x}\right) \tanh \left[\frac{E_{\eta}\left(k_{x}\right)}{2 k_{B} T}\right] .
$$

In the reduced low-dimensional system, we may define a spectral function depending on the site and partial momentum $A_{\underline{\mathbf{i}} \sigma}(\mathbf{k}, \sigma)$ as

$$
A_{\underline{\mathbf{i}}}(\mathbf{k}, \omega)=\sum_{\eta, \sigma} \frac{u_{\underline{\mathbf{i}} \sigma}^{\eta}(\mathbf{k})^{2}}{\omega-E_{\eta}(\mathbf{k})+i \Gamma} .
$$

The normal state Hamiltonian [Eq. (2)] can be expressed in the momentum space by a full Fourier transformation, which is written as the $2 \times 2$ matrix,

$H_{t}=\left(h-2 \sum_{\alpha} t_{\alpha} \cos k_{\alpha}\right) \sigma_{z}+2 \lambda\left(\sin k_{x} \sigma_{x}+\sin k_{y} \sigma_{y}\right)-\mu \sigma_{0}$.

Here $\sigma_{0}$ is the identity matrix and $\sigma_{x, y, z}$ are the Pauli matrices. The two normal state energy bands are given by

$E(\mathbf{k})$

$$
=\mu \pm \sqrt{4 \lambda^{2}\left(\sin ^{2} k_{x}+\sin ^{2} k_{y}\right)+\left(h-2 \sum_{\alpha} t_{\alpha} \cos k_{\alpha}\right)^{2}} .
$$

With $\mu=0$, the system may enter a Weyl semimetal phase. A pair of Weyl points $W_{ \pm}$may be obtained from the above energy bands through choosing appropriate parameters, with $W_{ \pm}=\left[0,0, \pm \arccos \left(\frac{h-2 t_{x}-2 t_{y}}{2 t_{z}}\right)\right]$.

In the present work, the parameters are chosen as $t_{x}=t_{y}=$ $0.5, t_{z}=1, \lambda=0.5$, and $h=2+2 \cos (\pi / 4)$. In this case, there exist two Weyl points at $(0,0, \pm \pi / 4)$. If not specially mentioned, hereafter, the chemical potential is set as $\mu=0.5$, then the system is metallic with a Fermi pocket surrounding each Weyl point. We have checked numerically that our main results are not sensitive to the parameters.

\section{RESULTS AND DISCUSSIONS}

We first study the normal state electronic structure by setting $\Delta_{\mathrm{ii}} \equiv 0$. The Hamiltonian in $k_{x}-y-k_{z}$ space is considered with open boundary condition along $y$ direction $(1 \leqslant y \leqslant$ 200). The zero-energy spectral functions $\left[A_{y}\left(k_{x}, k_{z}, \omega=0\right)\right]$ in the bulk and the system edge are presented in Figs. 1(a) and $1(\mathrm{~b})$, respectively. As is known, $A_{y}\left(k_{x}, k_{z}, 0\right)$ should be maximum at the Fermi momentum. Thus, the normal state Fermi surface is obtained from Fig. 1. In the bulk, as is seen in Fig. 1(a), there exists one Fermi pocket surrounding each Weyl point $\left(0,0, \pm Q_{z}\right)$, with the size of the pockets controlled by

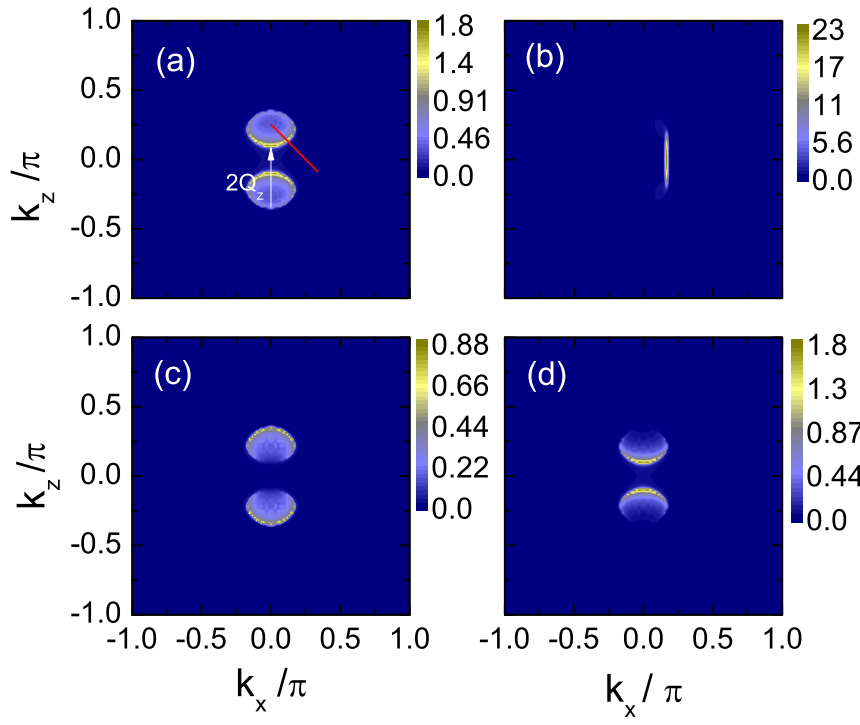

FIG. 1. Intensity plots of normal-state zero-energy spectral functions with open-boundary condition along $y$ direction $(1 \leqslant y \leqslant 200)$. (a) The spectral function in the bulk with $y=100$. The arrow indicates the Fermi surface nesting vector. (b) The spectral function at the system edge with $y=1$. (c) The spectral function of spin up particles with $y=100$. (d) The spectral function of spin down particles with $y=100$.

the chemical potential $\mu$. At the system edge, as is shown in Fig. 1(b), open Fermi arc forms, which is connecting the tips of the bulk Fermi pockets and is perpendicular to the $k_{x}$ axis. The spin-dependent zero-energy spectral functions $A(\mathbf{k}, \uparrow)$ and $A(\mathbf{k}, \downarrow)$ are plotted in Figs. 1(c) and 1(d), respectively. As is shown, significant spin imbalance exists: the spin-up quasiparticles have large spectral weight as $\left|k_{z}\right|>\pi / 4$, while the spin-down ones have large spectral weight as $\left|k_{z}\right|<\pi / 4$. And it seems that the Fermi surface nesting exists with the nesting vector $2 Q_{z}=\pi / 2$, as indicated in Fig. 1(a). From the above-presented fermiology, it is understandable that there may exist two competing superconducting states, i.e., the BCS-type pairing and the FFLO-type pairing with the net cooper pair momentum $\mathbf{Q}_{\mathbf{f}}=\left(0,0, \pm 2 Q_{z}\right)$.

Now let us study the superconducting state Hamiltonian. With the periodic boundary condition along $x$ direction the Hamiltonian can be expressed in the $k_{x}-y-z$ plane [Eqs. (10) and (11)]. The system size is set as $128 \times 32 \times 32$. The BdG equations are solved self-consistently, and the superconducting order parameters are obtained based on Eq. (12). As the solution is converged, only uniform superconducting order parameters are obtained; i.e., the BCS-type pairing is supported by a self-consistent calculation. Note that this result does not depend on the boundary condition along the $y$ and $z$ direction. Especially, with a periodic boundary condition, one may further transform the Hamiltonian to the momentum space for the $y$ direction. Thus, the numerical calculation for a much larger system size is possible. The size effect is checked numerically and the BCS solution is robust. We also consider different initial input order parameters: uniform ones (BCS-type), periodic ones (FFLO-type), and random ones. The converged solution is always uniform when the self-consistency is achieved. 


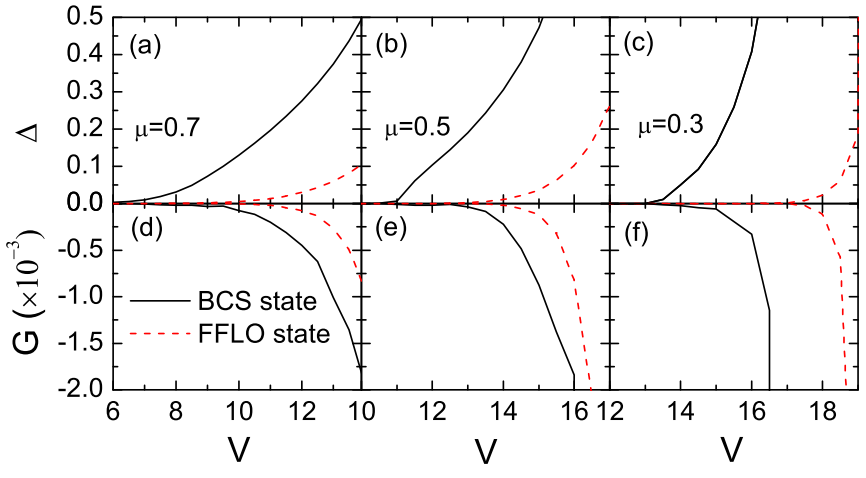

FIG. 2. The gap magnitude and the superconducting condensation energy in the BCS state and FFLO state, respectively.

By setting $\Delta_{\mathrm{ii}}=\Delta_{0}($ BCS-type $)$ or $\Delta_{\mathrm{ii}}=\Delta_{0} \cos \left(\mathbf{R}_{\mathbf{i}} \cdot \mathbf{Q}_{\mathbf{f}}\right)$ (FFLO-type), the gap magnitude $\Delta_{0}$ and the superconducting condensation energy with different pairing strength $V$ and chemical potential $\mu$ are plotted in Fig. 2. As is seen in Figs. 2(a)-2(c), for the BCS-type pairing, the gap magnitude is larger and the superconductivity occurs for relatively smaller pairing interaction, which indicates that the BCS pairing wins over the FFLO pairing. This conclusion is further confirmed through the condensation energy [Figs. 2(d)-2(f)]. As presented, the free energy of the BCS state is always lower than that of the FFLO state. This gives another strong evidence that the BCS state should be the ground state.

In the above-presented numerical results, the superconductivity appears for a strong bare attractive interaction $(V \geqslant 6)$. The system is in the metallic state for weaker interactions. This is significantly different from that seen in Ref. [17], where superconductivity can occur for a very weak interaction with $0<V_{0}<0.12 t$, while in Ref. [17], the electron is restricted within a thin shell around the Fermi surface. Thus, the required pairing attraction can be rather weak as long as the shell is sufficiently thin. However, the bare interaction should be rather stronger to achieve superconductivity based on the BdG approach employed here as all of the valence electrons are taken into account. A threshold pairing strength, above which the superconductivity occurs, is required. Usually, the threshold value depends on the size of the Fermi surface and the normal state bandwidth. It is insightful to compare the present results with those in other families of superconductors based on the BdG technique. For high- $\mathrm{T}_{c}$ superconductors, where the Fermi surface is rather large, the required pairing strength is relatively small. The gap magnitude is close to the experimental value with the pairing interaction $V$ being about $1 t \sim 2 t$ [30-33]. While for an effective model describing the topological superconductors, a larger pairing strength (about $4 t \sim 5 t)$ is required, because the normal state Fermi surface is usually much smaller than that of high- $\mathrm{T}_{c}$ superconducting materials [34,35]. For the Weyl superconductors, since the bandwidth is larger (about 15t) and the Fermi pockets are rather tiny, it is understandable that a rather strong bare interaction is required to achieve superconductivity. Notably, as seen from Fig. 2, the obtained gap magnitude is rather small; namely, the effective paring strength, which is a key and relevant coupling parameter for superconductivity, is weak, such that the real-space BdG employed here can still work.
It is also meaningful to discuss whether such a strong bare interaction is relevant to a real system. Note that here " $t_{\alpha}$ " in Eq. (1) is not the usual hopping constant. For real materials, it is a renormalized one, and thus its scale is unclear, which may need to be determined experimentally. On the other hand, such a strong bare interaction may be realized in cold atom systems, where the $s$-wave pairing has already been realized [28]. Moreover, as was proposed in Ref. [26], the strong attractive interaction can be realized in ultracold atom systems by tuning the system close to the Fechbach resonance.

We now discuss the competition between the BCS-type pairing and the FFLO one in more detail. In fact, in the mean-field level, the FFLO pairing is naturally realized by adding a Zeeman field term to the superconducting Hamiltonian $[29,31]$, while in topological superconductors, the FFLO pairing will be destroyed by the spin-orbital coupling [29]. In Weyl superconductors, the normal state electronic structure is much more complicated, and thus it is not so easy to draw a conclusion intuitively. In Ref. [17], it was indicated that an FFLO-type superconducting should be a most favorable state. In their calculations, the superconducting pairing is considered merely at a thin shell around the Fermi surface. It seems that the appearance of the FFLO state could indeed be understood based on the Fermi surface topology.

In fact, the approximation by only taking into account the electrons near the Fermi surface is reasonable based on the BCS theory. As is known, usually for a metal, the mixture of the electrons and holes is negligibly weak when away from the Fermi surface. As a result, the superconducting pairing is determined entirely by the Fermi surface topology.

However, it is needed to emphasize that here the Fermi surface analysis is not enough to confirm the superconducting pairing. First, due to the lattice distortion, actually one cannot restrict strictly the electrons within a sphere shell for the FFLO pairing. Thus, to study the competition between the two superconducting pairings based on the lattice-type model, a method beyond the Fermi surface analysis is demanded. Second, we can estimate the volume of the two Fermi pockets from Fig. 1 or Eq. (15). It is only about $0.35 \%$ of that of the Brillouin zone. Thus, for the present case, the Fermi pockets are very tiny and the finite-energy quasiparticles may be also important to determine the ground states. At last, the normal state Hamiltonian is a two-band model with one hole band and one electron band. Thus, the mixture of the electrons and holes may be still considerably strong for the finite energy. Thus, it is important to investigate the finite-energy band structure to draw a more definite conclusion for the superconducting pairing.

Let us study the finite-band structure of the BCS and FFLO superconducting pairing states. These two states are illustrated in Fig. 3(a). For BCS pairing state, the quasiparticles momenta from one pair are $\mathbf{k}$ and $-\mathbf{k}$, respectively. And for the FFLO state, the momenta are $\mathbf{k}$ and $-\mathbf{k}+\mathbf{Q}_{\mathbf{f}}$, respectively. So more conclusive results may be obtained by comparing the spin-dependent energy bands at the momenta $\mathbf{k},-\mathbf{k}$, and $-\mathbf{k}+\mathbf{Q}_{\mathbf{f}}$. For the present model, the energy bands from the $\mathrm{BdG}$ equations are usually the superpositions of the spin-up and spin-down electrons due to the spin-flip term. It is more insightful to study the spectral functions to obtain the spin-dependent quasiparticle energies. 

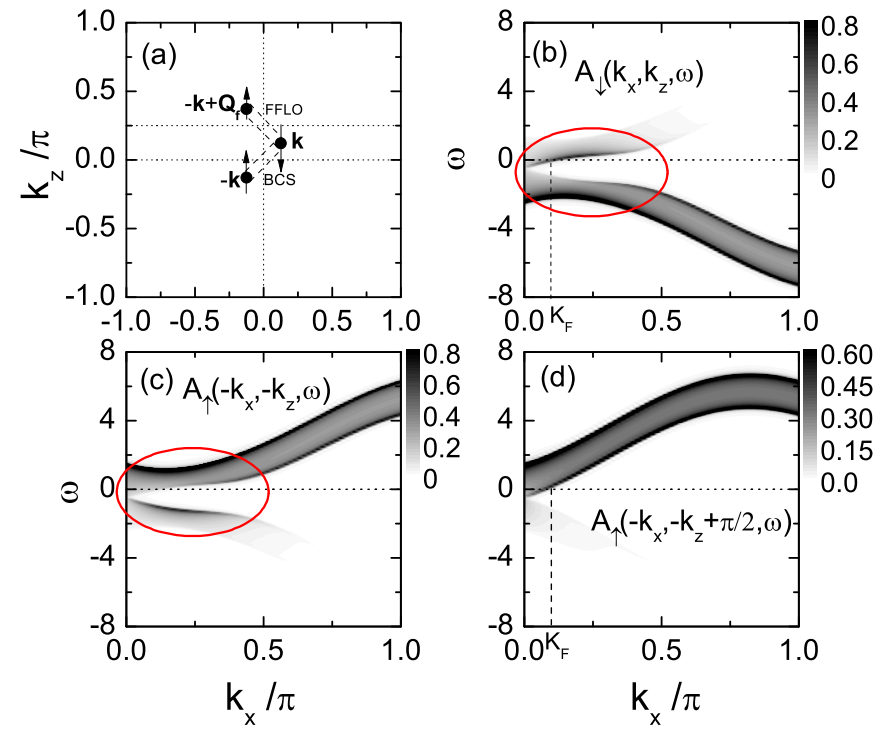

FIG. 3. (a) Schematic illustration of the BCS pairing and FFLO pairing, respectively. (b)-(d) The spin-dependent spectral functions as functions of the energy and momentum with $k_{z}=\pi / 4-k_{x}$ [along the (red) solid line cut shown in Fig. 1(a)].

The spectral function for the spin-down quasiparticles as functions of the energy and momentum $\left[A_{\downarrow}(\mathbf{k}, \omega)\right]$ is plotted in Fig. 3(b). The corresponding spectral functions for spin-up quasiparticles at the momenta $-\mathbf{k}$ and $-\mathbf{k}+\mathbf{Q}_{\mathbf{f}}$ are presented in Figs. 3(c) and 3(d), respectively. The two energy bands are seen clearly. Especially, the spectral weight of the two bands are considerably strong for a large energy range [as indicated by (red) circles in Figs. 3(b) and 3(c)]. This also proposes that the Fermi surface topology analysis is not enough to determine the ground state. We compare Figs. 3(b) with 3(c) to roughly evaluate whether the BCS pairing is favorable. At low energies, as displayed, the energy bands at $\mathbf{k}$ and $-\mathbf{k}$ are obviously similar. The quasiparticle velocities are small near the Fermi energy from both spectra. The increase of the density of states for low-energy quasiparticles may increase the possibility of the BCS pairing. We then compare Figs. 3(b) with 3(d) to study the possibility of the FFLO pairing. As is seen, the Fermi momenta $K_{F}$ are nearly the same. This feature favors the FFLO pairing. While, on the other hand, their quasiparticle band dispersions are significantly different, that is, the states at $-\mathbf{k}+\mathbf{Q}_{\mathbf{f}}$ and $\mathbf{k}$ have different energies when away from the Fermi surface. This feature is unfavorable to the FFLO pairing. As a result, when considering the quasiparticle pairing in the whole Brillouin zone, the BCS pairing wins over the FFLO pairing.

We may get a further insight from the Fermi surface analysis for the unfavorable FFLO pairing. With the net cooper pair momentum $\mathbf{Q}_{\mathbf{f}}$, the upper Fermi pocket is fully gapped. However, for this kind of pairing, the lower Fermi surface is not entirely gapped; i.e., when $\mathbf{k}$ is near the lower Fermi pocket, the electron with the momentum $-\mathbf{k}+\mathbf{Q}_{\mathbf{f}}$ is rather far away from the Fermi surface. The two electrons from one pair have significantly different energies. This kind of pairing may increase the free energy. There exists another pairing channel with the net momentum $-\mathbf{Q}_{\mathbf{f}}$, for which the lower
Fermi pocket is gapped. While for this pairing channel, the upper Fermi surface is completely not gapped. When these two pairing channels coexist, the system is fully gapped. While a lot of Cooper pairs are formed by the electrons with different energies. This kind of pairing is actually neglected in Ref. [17] because the electrons are restricted around the Fermi surface there. While it indeed strains the FFLO pairing. On the other hand, in Ref. [17], the lattice distortion is neglected due to the low-energy effective continuous approximation. Actually, in the lattice system, the FFLO pairing state is strained further. Based on the lattice-type model the Fermi pockets are surely not symmetric exactly with respect to the Weyl nodes. Such asymmetric feature may also suppress the FFLO-type pairing.

Very recently, it was also proposed that BCS-type pairing should win out for an inversion-symmetric doped Weyl semimetal system [24]. This is consistent with our above analysis. While in Ref. [24], the odd-parity pairing state is revealed, consistent with a previous study [20]. Note that the lattice-type model is considered in the present work, qualitatively different from those in Refs. [20,24]. For a lattice model, the odd parity pairing would obviously not occur for the on-site pairing potential. The superconductivity in the odd-parity channel may be studied by taking into account a nearest-neighbor pairing potential. This is an interesting issue and may require further investigation.

We now study the physical properties in the BCS-type superconducting state with the uniform pairing order parameter $\Delta_{\mathrm{ii}} \equiv \Delta_{0}$. As discussed in Refs. [16,17], the system has gapless nodes along the $k_{z}$ axis. In detail, if the condition $\left|h-2 t_{x}-2 t_{y} \pm \sqrt{\mu^{2}+\Delta_{0}^{2}}\right|<2 t_{z}$ is satisfied, there are four nodes at points $\left(0,0, \pm Q_{ \pm}\right)$, with

$$
Q_{ \pm}=\arccos \frac{h-2 t_{x}-2 t_{y} \pm \sqrt{\mu^{2}+\Delta_{0}^{2}}}{2 t_{z}} .
$$

Here the nodes are inherited from the initial Fermi pockets. Therefore, the nodes at $\left(0,0, Q_{ \pm}\right)$have the same chirality, while the chirality of the nodes at $\left(0,0,-Q_{ \pm}\right)$are opposite. As the chemical potential $\mu$ or the pairing order $\Delta_{0}$ increases, the topologically protected nodes with opposite chirality may annihilate in pairs $[36,37]$. Thus, a pair of nodes at $\left(0,0, \pm Q_{+}\right)$ may disappear and only the nodes $\left(0,0, \pm Q_{-}\right)$are left. As a result, there may exist two different $\mathrm{BCS}$ states, named as BCSI state with four bulk nodes and BCS-II state with two bulk nodes. Further increasing the pairing strength, the nodes $\pm Q_{-}$ may also disappear while this may require unreasonably large superconducting order parameter. This case is not considered in the present work.

The electronic structure in BCS-I state has been discussed previously $[24,25]$. We would like to discuss this state in more detail. Here the BCS-I state is achieved through considering the relatively small chemical potential and the pairing order parameter with $\mu=0.5$ and $\Delta_{0}=0.2$. This gives $Q_{+}$and $Q_{-}$ to be $0.07 \pi$ and $0.36 \pi$, respectively. By considering the open boundary condition along the $y$ direction and transforming the Hamiltonian to the momentum space along $x$ and $z$ directions, the quasiparticle energy spectra along $k_{x}=0$ is presented in Fig. 4(a). This band structure is similar to that obtained by previous calculations [24,25]. There exists two segments of 

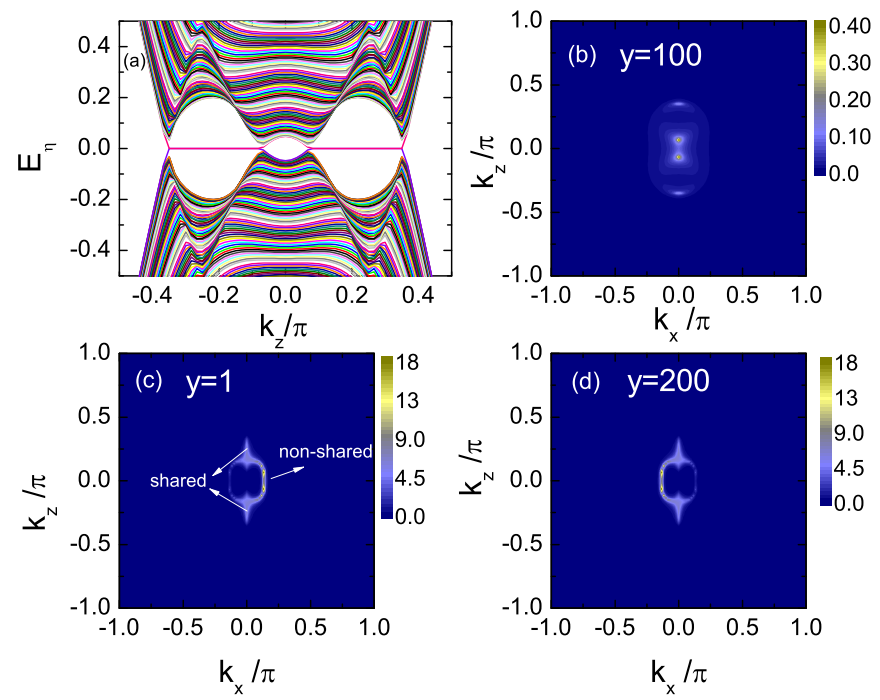

FIG. 4. Numerical results of the BCS-I state. (a) The eigenvalues of the Hamiltonian along $k_{x}=0$. (b)-(d) Intensity plots of zero energy spectral functions.

Fermi arcs connecting nodal points $Q_{+}$and $Q_{-}$, indicating the presence of the edge states. The zero-energy spectral functions in the bulk and system edges are plotted in Figs. 4(b)-4(d). As is seen, in the bulk four Fermi points at the momentum $k_{z}= \pm Q_{ \pm}$exist, consistent with our above analysis and the band structure shown in Fig. 4(a). At the system edges, there exist several segments of Fermi arcs. Especially, the Fermi arcs connecting the Fermi points $\left(0, Q_{-}\right)$and $\left(0, Q_{+}\right)$[or $\left(0,-Q_{-}\right)$ and $\left.\left(0,-Q_{+}\right)\right]$have the same spectral weight at the two-system boundary, as presented in Figs. 4(c) and 4(d). Such shared edge states is similar to that of the Majorana bound states and one zero-energy quasiparticle may be decoupled by two spatially separated ones. At the momentum $-Q_{+}<k_{z}<Q_{+}$, there exist nonshared edge states, with $k_{x}<0$ and $k_{x}>0$ parts belong to different boundaries.

It is important to pinpoint that there is no separate MFs in the BCS-I state, even when the shared edge states exist. Usually, two MFs $\gamma_{1,2}$ can be obtained from one zero mode $C=$ $u_{k} \psi^{\dagger}(k)+v_{-k} \psi(-k)$ with $\gamma_{1}=C+C^{\dagger}$ and $\gamma_{2}=i\left(C-C^{\dagger}\right)$. For usual topological superconductors, generally the edge states occur at the high-symmetric points, i.e., $k=0$ or $k=\pi$. As a result, $k$ and $-k$ are equivalent points. Then $\gamma_{1}$ and $\gamma_{2}$ are naturally decoupled and locating at different boundaries. While in the present BCS-I case, it is obvious that one cannot obtain the separate $\gamma_{1,2}$ through the above procedure.

We now turn to look into the properties of the BCS-II state. Generally, this state can be achieved by increasing the chemical potential $\mu$. For a large chemical potential, the two Fermi pockets may merge into a single one. As a result, only two bulk nodes exist in the superconducting state. We here consider the chemical potential $\mu=0.7$, which corresponds to the case of two bulk nodes $\left(0,0, \pm Q_{-}\right)$with $Q_{-}=0.39 \pi$. The numerical results for this state are presented in Fig. 5. The energy spectrum along $k_{x}=0$ is plotted in Fig. 5(a). The zero-energy spectral functions in the bulk and at system edge are presented in Figs. 5(b) and 5(c), respectively. From Fig. 5(a), there are two nodal points in the bulk spectrum and one segment

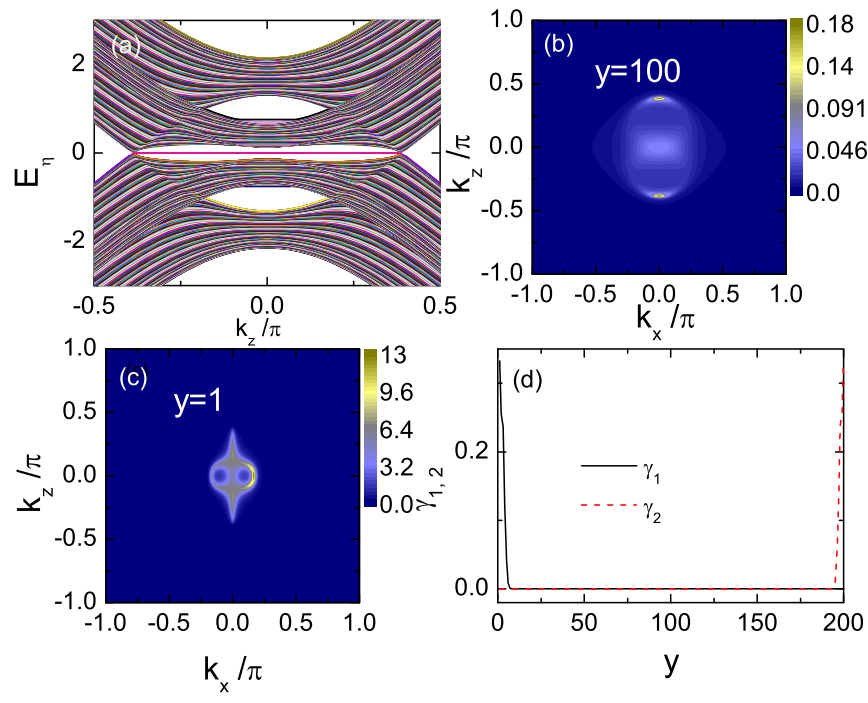

FIG. 5. Numerical results of the BCS-II state. (a) The eigenvalues of the Hamiltonian along $k_{x}=0$. Panels (b) and (c) are intensity plots of zero-energy spectral functions at the system bulk and edge, respectively. (d) The spatial distributions of the two MFs at the momentum $\left(k_{x}, k_{z}\right)=(0,0)$.

of Fermi arc connecting $Q_{-}$and $-Q_{-}$at the system edge. Here the Fermi arc is shared by the two system boundaries. A significant different result from the BCS-I state is that there is a zero-energy edge state at the momentum $(0,0)$ point, giving the possibility of the MFs excitation. The existence of the MFs excitation is further confirmed numerically. The spatial distribution of the two MFs from the zero-energy fermion at $(0,0)$ point is displayed in Fig. 5(d). As is seen, two MFs are completely separated and locate at the two system edges.

At last we would like to look into the FFLO-type pairing state. Although the FFLO-type pairing is not supported by our self-consistent calculation. While it is still a potential pairing state and may appear upon some additional interaction. The FFLO-type pairing is denoted as

$$
H_{\mathrm{FFLO}}=\sum_{\mathbf{i}}\left(\Delta_{1} e^{i \mathbf{Q}_{\mathbf{f}} \cdot \mathbf{R}_{\mathbf{i}}} c_{\mathbf{i} \uparrow}^{\dagger} c_{\mathbf{i} \downarrow}^{\dagger}+\Delta_{2} e^{-i \mathbf{Q}_{\mathbf{f}} \cdot \mathbf{R}_{\mathbf{i}}} c_{\mathbf{i} \uparrow}^{\dagger} c_{\mathbf{i} \downarrow}^{\dagger}+\text { H.c. }\right) .
$$

The above Hamiltonian can be rewritten in the momentum space,

$$
H_{\mathrm{FFLO}}=\sum_{\mathbf{k}}\left(\Delta_{1} c_{\mathbf{k} \uparrow}^{\dagger} c_{-\mathbf{k}+\mathbf{Q}_{\mathbf{f}} \downarrow}^{\dagger}+\Delta_{2} c_{\mathbf{k} \uparrow}^{\dagger} c_{-\mathbf{k}-\mathbf{Q}_{\mathbf{r}} \downarrow}^{\dagger}+\text { H.c. }\right) .
$$

Taking into account the inversion symmetry, we here consider the LO state with $\Delta_{1}=\Delta_{2}=0.2$.

The numerical results for the FFLO state are presented in Fig. 6. The quasiparticle energies along $k_{x}=0$ is plotted in Fig. 6(a). There exist two zero-energy states at the momentum $k_{z}= \pm \pi / 4$. The bulk-energy spectrum is fully gapped and no Fermi surface exists. The zero-energy spectral functions at the system edges are displayed in Figs. 6(b) and 6(c), respectively. The open Fermi arcs exist at the system edges. The Fermi arcs are not shared by the two boundaries, except for the points $\pm \mathbf{k}_{\mathbf{0}}$ with $\mathbf{k}_{\mathbf{0}}=(0, \pi / 4)$, which are the crossing points of the 

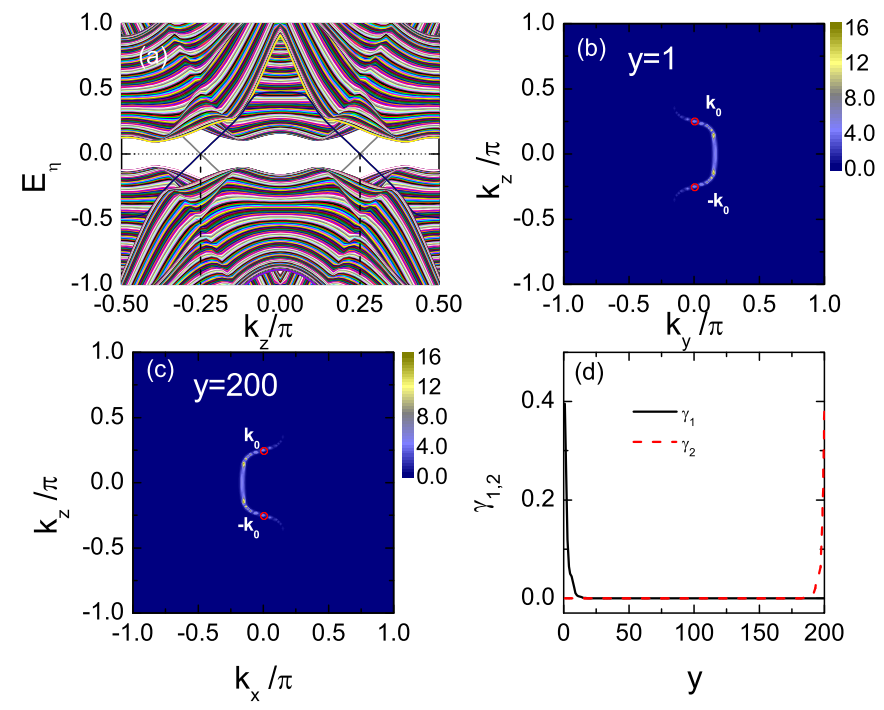

FIG. 6. Numerical results of the FFLO-type pairing. (a) The eigenvalues of the Hamiltonian along $k_{x}=0$. (b) and (c) Intensity plots of zero energy spectral functions at two boundaries, respectively. (d) The spatial distributions of the two MFs at the momentum $\mathbf{k}_{\mathbf{0}}$.

two Fermi arcs. At the points $\pm \mathbf{k}_{\mathbf{0}}$, the quasiparticles have the same spectral weight at the two boundaries. Thus, the MFs excitation may occur at these two points. Here $\mathbf{k}_{\mathbf{0}}$ and the net Cooper pair momentum $\mathbf{Q}_{\mathbf{f}}$ satisfies $\mathbf{k}_{\mathbf{0}} \equiv \mathbf{Q}_{\mathbf{f}} / 2$. This is an important relation. It is robust and does not change upon parameters.

The shared edge state at the momentum $\mathbf{k}_{\mathbf{0}}$ is essential to produce MFs. In the FFLO state, the quasiparticles can be expressed as $C=u_{\mathbf{k}} \psi^{\dagger}(\mathbf{k})+v_{-\mathbf{k}+\mathbf{Q}_{\mathbf{f}}} \psi\left(-\mathbf{k}+\mathbf{Q}_{\mathbf{f}}\right)$. At the point $\mathbf{k}=\mathbf{k}_{\mathbf{0}}$, the quasiparticle is expressed as $C=u_{\mathbf{k}_{\mathbf{0}}} \psi^{\dagger}\left(\mathbf{k}_{\mathbf{0}}\right)+$ $v_{\mathbf{k}_{\mathbf{0}}} \psi\left(\mathbf{k}_{\mathbf{0}}\right)$. Obviously, the quasiparticle is a MF if the condition $u_{\mathbf{k}_{0}}=v_{\mathbf{k}_{0}}^{*}$ satisfies. Actually, one can obtain two MFs $\gamma_{1,2}$ according to the standard method. Our numerical results verify that two separate MFs indeed exist. The numerical result for the spatial distribution of $\gamma_{1,2}$ is plotted in Fig. $6(\mathrm{~d})$. As is seen, two MFs locate at two boundaries of the system.

\section{SUMMARY}

In summary, we have studied theoretically the superconductivity in inversion-symmetric doped Weyl semimetals based on the BdG equations. Our self-consistent calculations have indicated that the BCS-like pairing may be more favorable than the FFLO pairing. The competition between the BCS-like pairing and FFLO pairing is discussed based on the Fermi surface topology and finite-energy band structure. The physical properties of the BCS-like pairing and FFLO pairing states have also been addressed. Two different BCS states, named BCS-I state and BCS-II state, are revealed. There are four bulk nodes in BCS-I state and no MFs exists in this state. For the BCS-II state, the number of bulk nodes reduces to two and the MFs excitation occur in this state. For the FFLO state, the energy spectrum is full gapped. The open Fermi arc appears at the system edge. The separate MFs exist at the system boundaries.

\section{ACKNOWLEDGMENTS}

We thank D. B. Zhang and Y. X. Zhao for helpful discussions. This work was supported by the NSFC (Grant No. 11374005), the NCET (Grant No. NCET-12-0626), the GRF (Grants No. HKU173051/14P and No. HKU173055/15P), and the CRF (Grant No. HKU8/11G) of Hong Kong.
[1] X.-L. Qi and S.-C. Zhang, Rev. Mod. Phys. 83, 1057 (2011).

[2] X. Wan, A. M. Turner, A. Vishwanath, and S. Y. Savrasov, Phys. Rev. B 83, 205101 (2011).

[3] Y. X. Zhao and Z. D. Wang, Phys. Rev. Lett. 110, 240404 (2013).

[4] Y. X. Zhao and Z. D. Wang, Phys. Rev. Lett. 114, 206602 (2015).

[5] H. Weng, C. Fang, Z. Fang, A. Bernevig, and X. Dai, Phys. Rev. X 5, 011029 (2015).

[6] S.-M. Huang, S.-Y. Xu, I. Belopolski, C.-C. Lee, G. Chang, B. Wang, N. Alidoust, G. Bian, M. Neupane, C. Zhang, S. Jia, A. Bansil, H. Lin, and M. Zahid Hasan, Nat. Commun. 6, 7373 (2015).

[7] S.-Y. Xu, I. Belopolski, N. Alidoust, M. Neupane, G. Bian, C. Zhang, R. Sankar, G. Chang, Z. Yuan, C.-C. Lee, S.-M. Huang, H. Zheng, J. Ma, D. S. Sanchez, B. Wang, A. Bansil, F. Chou, P. P. Shibayev, H. Lin, S. Jia, and M. Z. Hasan, Science 349, 613 (2015).

[8] B. Q. Lv, H. M. Weng, B. B. Fu, X. P. Wang, H. Miao, J. Ma, P. Richard, X. C. Huang, L. X. Zhao, G. F. Chen, Z. Fang, X. Dai, T. Qian, and H. Ding, Phys. Rev. X 5, 031013 (2015).

[9] S.-Y. Xu, N. Alidoust, I. Belopolski, Z. Yuan, G. Bian, T.-R. Chang, H. Zheng, V. N. Strokov, D. S. Sanchez, G. Chang, C. Zhang, D. Mou, Y. Wu, L. Huang, C.-C. Lee, S.-M. Huang,
B. Wang, A. Bansil, H.-T. Jeng, T. Neupert, A. Kaminski, H. Lin, S. Jia, and M. Zahid Hasan, Nat. Phys. 11, 748 (2015).

[10] N. Xu, H. M. Weng, B. Q. Lv, C. Matt, J. Park, F. Bisti, V. N. Strocov, D. gawryluk, E. Pomjakushina, K. Conder, N. C. Plumb, M. Radovic, G. Auts, O. V. Yazyev, Z. Fang, X. Dai, G. Aeppli, T. Qian, J. Mesot, H. Ding, and M. Shi, arXiv:1507.03983.

[11] S.-Y. Xu, I. Belopolski, D. S. Sanchez, C. Zhang, G. Chang, C. Guo, G. Bian, Z. Yuan, H. Lu, T.-R. Chang, P. P. Shibayev, M. L. Prokopovych, N. Alidoust, H. Zheng, C.-C. Lee, S.-M. Huang, R. Sankar, F. Chou, C.-H. Hsu, H.-T. Jeng, A. Bansil, T. Neupert, V. N. Strocov, H. Lin, S. Jia, and M. Z. Hasan, Sci. Adv. 1, e1501092 (2015).

[12] Z. Wang, Y. Zheng, Z. Shen, Y. Zhou, X. Yang, Y. Li, C. Feng, and Z.-A. Xu, arXiv:1506.00924.

[13] A. A. Soluyanov, D. Gresch, Z. Wang, Q. Wu, M. Troyer, X. Dai, and B. A. Bernevig, Nature (London) 527, 495 (2015).

[14] Y. Sun, S.-C. Wu, M. N. Ali, C. Felser, and B. Yan, Phys. Rev. B 92, 161107 (2015).

[15] Y. Qi, P. G. Naumov, M. N. Ali, C. R. Rajamathi, O. Barkalov, M. Hanfland, S.-C. Wu, C. Shekhar, Y. Sun, V. Süß, M. Schmidt, E. Pippel, P. Werner, R. Hillebrand, T. Förster, E. Kampertt, W. Schnelle, S. Parkin, R. J. Cava, C. Felser, B. Yan, and S. A. Medvedev, arXiv:1508.03502. 
[16] T. Meng and L. Balents, Phys. Rev. B 86, 054504 (2012).

[17] G. Y. Cho, J. H. Bardarson, Y.-M. Lu, and J. E. Moore, Phys. Rev. B 86, 214514 (2012).

[18] T. Das, Phys. Rev. B 88, 035444 (2013).

[19] W. Chen, L. Jiang, R. Shen, L. Sheng, B. G. Wang, and D. Y. Xing, Europhys. Lett. 103, 27006 (2013).

[20] H. Wei, S.-P. Chao, and V. Aji, Phys. Rev. B 89, 014506 (2014).

[21] U. Khanna, A. Kundu, S. Pradhan, and S. Rao, Phys. Rev. B 90, 195430 (2014).

[22] S. A. Yang, H. Pan, and F. Zhang, Phys. Rev. Lett. 113, 046401 (2014).

[23] B. Liu, X. Li, L. Yin, and W. V. Liu, Phys. Rev. Lett. 114, 045302 (2015).

[24] G. Bednik, A. A. Zyuzin, and A. A. Burkov, Phys. Rev. B 92, 035153 (2015).

[25] B. Lu, K. Yada, M. Sato, and Y. Tanaka, Phys. Rev. Lett. 114, 096804 (2015).

[26] S.-K. Jian, Y.-F. Jiang, and H. Yao, Phys. Rev. Lett. 114, 237001 (2015)

[27] C. Nayak, S. H. Simon, A. Stern, M. Freedman, S. D. Sarma, Rev. Mod. Phys. 80, 1083 (2008).
[28] T. Bourdel, L. Khaykovich, J. Cubizolles, J. Zhang, F. Chevy, M. Teichmann, L. Tarruell, S. J. J. M. F. Kokkelmans, and C. Salomon, Phys. Rev. Lett. 93, 050401 (2004); J. K. Chin, D. E. Miller, Y. Liu, C. Stan, W. Setiawan, C. Sanner, K. Xu, and W. Ketterle, Nature (London) 443, 961 (2006).

[29] T. Zhou, Y. Gao, and Z. D. Wang, Sci. Rep. 4, 5218 (2014).

[30] Y. Chen, Z. D. Wang, and C. S. Ting, Phys. Rev. B 67, 220501(R) (2003); Y. Chen and C. S. Ting, Phys. Rev. Lett. 92, 077203 (2004).

[31] T. Zhou and C. S. Ting, Phys. Rev. B 80, 224515 (2009).

[32] Y. Gao, H.-X. Huang, C. Chen, C. S. Ting, and W.-P. Su, Phys. Rev. Lett. 106, 027004 (2011).

[33] T. Zhou, D. Zhang, and C. S. Ting, Phys. Rev. B 81, 052506 (2010); T. Zhou, Z. D. Wang, Y. Gao, and C. S. Ting, ibid. 84, 174524 (2011).

[34] T. Zhou and Z. D. Wang, Phys. Rev. B 88, 155114 (2013).

[35] T. Zhou, X.-J. Li, Y. Gao, and Z. D. Wang, Phys. Rev. B 91, 014512 (2015).

[36] H. B. Nielsen and M. Ninomiya, Phys. Lett. B 105, 219 (1981).

[37] Y. X. Zhao and Z. D. Wang, Phys. Rev. Lett. 116, 016401 (2016). 